\title{
Parechovirus $A$ prevalence in adults in The Netherlands
}

\author{
Lieke Brouwer $^{1}\left[\right.$ D $\cdot$ Katja C. Wolthers ${ }^{1} \cdot$ Dasja Pajkrt $^{2}$
}

Received: 6 September 2019 / Accepted: 9 January 2020 / Published online: 14 February 2020

(c) The Author(s) 2020

\begin{abstract}
Human parechoviruses $(\mathrm{HPeV})$ of the species Parechovirus $A$ are highly prevalent disease-causing pathogens in children worldwide. HPeVs are capable of causing severe disease in adults as well, but the prevalence in adults may be much lower. The aim of our present study was to determine the prevalence of $\mathrm{HPeV}$ in clinical samples from adults sent in for diagnostic procedures in a tertiary hospital in the Netherlands. From a total of 10,645 samples obtained from 6175 patients, 20 samples from 11 patients $(0.18 \%)$ tested positive for HPeV by RT-PCR. Two patients were positive for HPeV-1, two for HPeV-3, and one for HPeV-6. Six HPeVs could not be typed. Eight of the $11 \mathrm{HPeV}$-positive patients were immunocompromised. Due to comorbidity, we were unable to attribute the patients' clinical symptoms to the HPeV infection. The HPeV prevalence in adults found in this study is low compared to $\mathrm{HPeV}$ prevalence in children. This may be largely explained by the high seropositivity rates in adults, although there could be other mechanisms involved.
\end{abstract}

\section{Introduction}

Human parechoviruses (HPeV) of the species Parechovirus $A$ of the family Picornaviridae are known to circulate extensively in children worldwide [1]. HPeVs can cause mild respiratory or gastrointestinal symptoms, as well as severe neurological disease, such as meningitis and encephalitis. Overall, the clinical relevance of $\mathrm{HPeV}$ infections in adults remains largely unknown. Several studies have found a very low $\mathrm{HPeV}$ prevalence in adults compared to children [2-6],

Handling Editor: Tim Skern.

Katja C. Wolthers and Dasja Pajkrt Both authors contributed equally to this work.

Electronic supplementary material The online version of this article (https://doi.org/10.1007/s00705-020-04547-0) contains supplementary material, which is available to authorized users.

Lieke Brouwer

lieke.brouwer@amsterdamumc.nl

1 Laboratory of Clinical Virology, Department of Medical Microbiology, Amsterdam University Medical Centers, Location Academic Medical Center, University of Amsterdam, Meibergdreef 9, 1105 AZ Amsterdam, The Netherlands

2 Department of Pediatric Infectious Diseases, Emma Children's Hospital, Amsterdam University Medical Centers, Location Academic Medical Center, University of Amsterdam, Amsterdam, The Netherlands although cases of adults experiencing severe illness caused by $\mathrm{HPeV}$ have been described [7-10]. We performed a retrospective study to identify the $\mathrm{HPeV}$ prevalence in adults in a tertiary hospital in Amsterdam, The Netherlands, and to determine the clinical relevance of these viruses in adults.

\section{Materials and methods}

Between January 1, 2008 and January 1, 2018, a total of 10,645 clinical samples from 6175 patients older than 18 years of age were tested for $\mathrm{HPeV}$ at the Amsterdam UMC, location AMC, a tertiary academic hospital in Amsterdam, The Netherlands. The samples included i.a. stool samples, nose/throat swabs, cerebrospinal fluid, and serum samples from patients with a variety of symptoms, including gastrointestinal, respiratory and neurological symptoms, and fever. The patients were either hospitalized or presented to the outpatient clinic of the AMC. The samples also included fecal samples from 93 healthy fecal donors. For all respiratory and stool samples, an in-house multiplex RT-PCR, including the HPeV 5'UTR, was performed routinely. Other samples were tested for $\mathrm{HPeV}$ when requested by the treating physician. For all positive samples, RNA was extracted [11], the viral protein 1 (VP1) region was amplified by a nested PCR, and a sequencing reaction was performed using a Big Dye Terminator Kit [12]. For the current study, the VP1 sequences were aligned with Mafft 
version 7 software [13], and a maximum-likelihood (ML) phylogenetic tree of the clinical strains and reference strains from the GenBank database was constructed using RAxML version 8.2.12 [14].

\section{Results}

Of the 10,645 samples, 20 samples $(0.19 \%)$, belonging to a total of ten patients and one healthy fecal donor without symptoms $(11 / 6,175,0.18 \%)$, tested positive for HPeV RNA. Information on gender, age and symptoms was recorded for all patients (Table 1). HPeV-positive patients reported respiratory, gastrointestinal and neurological symptoms, and fever. Out of the eleven cases, eight had an immunocompromised status due to (treatment for) hematologic malignancies.

Of the eleven viral strains, two could be typed as HPeV1, two as HPeV-3, and one as HPeV-6, while six could not be typed (Fig. 1). One patient had multiple positive stool samples over a period of over three months, one patient

Table 1 Characteristics and $\mathrm{HPeV}$ types of 11 patients with $\mathrm{HPeV}$-positive samples

\begin{tabular}{ll}
\hline & N/total \\
\hline Gender & \\
Male & $6 / 11$ \\
Female & $5 / 11$ \\
Age & \\
30-39 & $6 / 11$ \\
50-59 & $3 / 11$ \\
60-69 & $2 / 11$ \\
Symptoms & \\
Respiratory & $4 / 11$ \\
Gastrointestinal & $3 / 11$ \\
Neurological & $1 / 11$ \\
Fever & $4 / 11$ \\
Unknown & $1 / 11$ \\
Healthy & $1 / 11$ \\
Immunocompromised & \\
Yes & $8 / 11$ \\
No & $1 / 11$ \\
Unknown & $2 / 11$ \\
HPeV type & \\
HPeV-1 & \\
HPeV-3 & $2 / 11$ \\
HPeV-6 & $2 / 11$ \\
Unknown & $1 / 11$ \\
Sample type & $6 / 11$ \\
Ttool & \\
CSFroat swab & $6 / 11$ \\
Serum & $5 / 11$ \\
\hline & $1 / 11$ \\
& $1 / 11$ \\
\hline
\end{tabular}

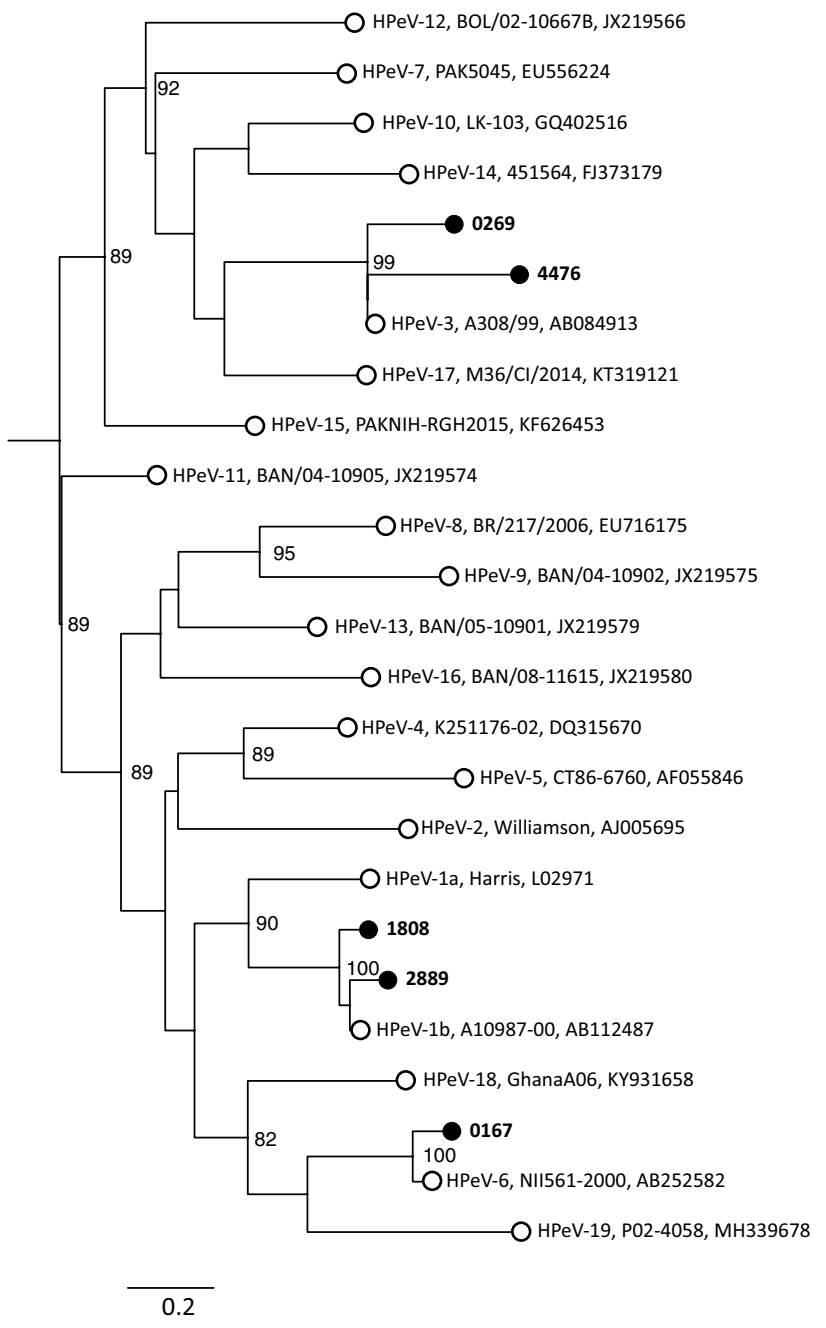

Fig. 1 Phylogenetic tree including the study strains (closed circles) and reference strains (open circles) from the GenBank database. Phylogenetic analysis was performed on the VP1 sequence (nt positions 2336-3037 of the genome sequence of the Harris HPeV-1 strain, accession no. L02791) using the maximum-likelihood (ML) method with the generalized time-reversible (GTR) + gamma nucleotide substitution model (1000 bootstrap replicates). For the reference strains, the type, strain name and accession numbers are given. Bootstrap values $\geq 70 \%$ for the branches are shown

tested $\mathrm{HPeV}$ positive in both throat swab and stool, and one patient tested HPeV positive in both serum and stool. All three patients were immunocompromised.

The HPeV sequences obtained in this study were deposited in the DDBJ/EMBL/GenBank nucleotide sequence databases with accession numbers MK861915 through MK861917. 


\section{Discussion}

We found a remarkably low $\mathrm{HPeV}$ prevalence of $0.18 \%$ among adults in our hospital. This is comparable to the low $\mathrm{HPeV}$ detection rates previously reported for critically ill adult patients in the Netherlands $(0.1 \%)$ [6] and adult cohorts in other countries $(0-0.3 \%)$ [2-5]. The low prevalence is in contrast with the $\mathrm{HPeV}$ prevalence found in children, which is much higher [1]. Furthermore, the low $\mathrm{HPeV}$ prevalence is in contrast with the enterovirus (EV) prevalence in adults, which is also higher [15-18]. The latter might be the result of the vast number of EV serotypes (>100), of which dozens are known to circulate on a large scale. It is possible that children do not encounter all circulating serotypes during childhood and remain susceptible to several EV types as an adult. For HPeVs, only types 1 and 3 are highly prevalent. Most children would gain immunity against both of those $\mathrm{HPeV}$ types at a very young age [19-21]. This humoral immunity is thought to be lifelong and would thus protect the vast majority of adults against $\mathrm{HPeV}$ infection.

There are additional mechanisms that might protect adults against infection with $\mathrm{HPeV}$. Although cross-immunity is not presumed to occur in EV infection, it could play a role in the immune response against HPeVs, as supported by data from previous studies [22]. In a cohort of mothers with $\mathrm{HPeV}$-infected children, we reported previously that an active infection was found in seven of 24 (29\%) mothers-a much higher prevalence than in our current study, indicating that $\mathrm{HPeV}$ infections do occur in adults [21]. A shorter period of viral shedding may explain why these $\mathrm{HPeV}$ infections in adults are often missed.

Notably, eight out of the $11 \mathrm{HPeV}$-positive individuals in our study were known to be immunocompromised. In two of the immunocompromised patients, the infection was disseminated, with multiple sample types testing positive for $\mathrm{HPeV}$. In one immunocompromised patient, the infection was prolonged, lasting for over three months. This suggests that immunocompromised individuals might be at a higher risk of $\mathrm{HPeV}$ infection, but also at a higher risk of experiencing an abnormal course of the infection. This could be a direct effect of compromised humoral immunity due to disease or treatment. Since most of our patients suffered from (severe) comorbidity, we cannot make any conclusions on the symptomatology of $\mathrm{HPeV}$ in these patients.

In conclusion, we found the prevalence of $\mathrm{HPeV}$ among adults in our hospital to be $0.18 \%$. The majority of the $\mathrm{HPeV}$-infected patients had a severe (humoral) immunodeficiency. This is in line with the current belief that protection against $\mathrm{HPeV}$ is mediated mainly through neutralizing antibodies, although other mechanisms may be involved in adults.
Funding This study did not receive any specific grant from funding agencies in the public, commercial or not-for-profit sectors.

\section{Compliance with ethical standards}

Conflict of interests None declared.

Ethical approval For our present study, no ethical approval was required.

Open Access This article is licensed under a Creative Commons Attribution 4.0 International License, which permits use, sharing, adaptation, distribution and reproduction in any medium or format, as long as you give appropriate credit to the original author(s) and the source, provide a link to the Creative Commons licence, and indicate if changes were made. The images or other third party material in this article are included in the article's Creative Commons licence, unless indicated otherwise in a credit line to the material. If material is not included in the article's Creative Commons licence and your intended use is not permitted by statutory regulation or exceeds the permitted use, you will need to obtain permission directly from the copyright holder. To view a copy of this licence, visit http://creativecommons.org/licenses/by/4.0/.

\section{References}

1. Brouwer L et al (2019) World-Wide prevalence and genotype distribution of Parechovirus A. Virol Retrovirol J 2(1):115

2. Masse S, Capai L, Falchi A (2017) Epidemiology of respiratory pathogens among elderly nursing home residents with acute respiratory infections in Corsica, France, 2013-2017. Biomed Res Int 2017:1423718. https://doi.org/10.1155/2017/1423718

3. Memish ZA et al (2015) Mass gathering and globalization of respiratory pathogens during the Hajj. Clin Microbiol Infect 21(6):57e1-57e18. https://doi.org/10.1016/j.cmi.2015.02.008

4. Parisi SG et al (2016) Viral infections of the central nervous system in elderly patients: a retrospective study. Int J Infect Dis 44:8-10. https://doi.org/10.1016/j.ijid.2016.01.012

5. Saikruang W et al (2014) Detection of diarrheal viruses circulating in adult patients in Thailand. Arch Virol 159(12):3371-3375. https://doi.org/10.1007/s00705-014-2191-3

6. van Someren Greve F et al (2018) Respiratory viruses in invasively ventilated critically Ill patients-a prospective multicenter observational study. Crit Care Med 46(1):29-36. https://doi. org/10.1097/CCM.0000000000002752

7. Chimunda T et al (2019) First reported case of human parechovirus encephalitis in an adult patient complicated by refractory status epilepticus. IDCases 15:e00475. https://doi.org/10.1016/j. idcr.2018.e00475

8. Kong KL et al (2017) Myocarditis caused by Human parechovirus in adult. Emerg Infect Dis 23(9):1571-1573. https://doi. org/10.3201/eid2309.161256

9. Mizuta K et al (2012) Epidemic myalgia in adults associated with Human parechovirus type 3 infection, Yamagata, Japan, 2008. Emerg Infect Dis 18(11):1787-1793. https://doi.org/10.3201/ eid1811.111570

10. Yamakawa T et al (2017) Clinical characteristics of 17 adult patients with epidemic myalgia associated with Human parechovirus type 3 infection. Rinsho Shinkeigaku 57(9):485-491. https ://doi.org/10.5692/clinicalneurol.cn-001035

11. Boom R et al (1990) Rapid and simple method for purification of nucleic acids. J Clin Microbiol 28(3):495-503 
12. Cremer J et al (2019) Highly sensitive parechovirus CODEHOP PCR amplification of the complete VP1 gene for typing directly from clinical specimens and correct typing based on phylogenetic clustering. J Med Microbiol. https://doi.org/10.1099/jmm.0.00097 4

13. Kyriakopoulou $\mathrm{Z}$ et al (2015) Recombination among human nonpolio enteroviruses: implications for epidemiology and evolution. Virus Genes 50(2):177-188. https://doi.org/10.1007/s1126 2-014-1152-y

14. Stamatakis A (2014) RAxML version 8: a tool for phylogenetic analysis and post-analysis of large phylogenies. Bioinformatics 30(9):1312-1313. https://doi.org/10.1093/bioinformatics/btu033

15. Fall A et al (2016) Enteroviruses and rhinoviruses: molecular epidemiology of the most influenza-like illness associated viruses in senegal. Am J Trop Med Hyg 95(2):339-347. https://doi. org/10.4269/ajtmh.15-0799

16. Kuramitsu $\mathrm{M}$ et al (2005) Non-polio enterovirus isolation among families in Ulaanbaatar and Tov province, Mongolia: prevalence, intrafamilial spread, and risk factors for infection. Epidemiol Infect 133(6):1131-1142. https://doi.org/10.1017/S095026880 5004139

17. Leitch EC et al (2009) Direct identification of Human enterovirus serotypes in cerebrospinal fluid by amplification and sequencing of the VP1 region. J Clin Virol 44(2):119-124. https://doi. org/10.1016/j.jcv.2008.11.015

18. Harvala $\mathrm{H}$ et al (2011) Comparison of Human parechovirus and enterovirus detection frequencies in cerebrospinal fluid samples collected over a 5-year period in edinburgh: HPeV type 3 identified as the most common picornavirus type. J Med Virol 83(5):889-896. https://doi.org/10.1002/jmv.22023

19. Karelehto E et al (2019) Seroepidemiology of parechovirus A3 neutralizing antibodies, Australia, The Netherlands, and United States. Emerg Infect Dis 25(1):148-152. https://doi.org/10.3201/ eid2501.180352

20. Westerhuis B et al (2013) Human parechovirus seroprevalence in Finland and The Netherlands. J Clin Virol 58(1):211-215. https ://doi.org/10.1016/j.jcv.2013.06.036

21. Karelehto E et al (2018) Human parechovirus 1, 3 and 4 neutralizing antibodies in Dutch mothers and infants and their role in protection against disease. Pediatr Infect Dis J 37(12):1304-1308. https://doi.org/10.1097/INF.0000000000001986

22. Westerhuis BM et al (2015) Human memory B cells producing potent cross-neutralizing antibodies against Human parechovirus: implications for prevalence, treatment, and diagnosis. J Virol 89(15):7457-7464. https://doi.org/10.1128/JVI.01079-15

Publisher's Note Springer Nature remains neutral with regard to jurisdictional claims in published maps and institutional affiliations. 